\title{
Research of digital temperature measurement system in vacuum thermal test based on DS18B20
}

\author{
Zhu Xi, An Wangqing, Liu Chang, Li ZhenWei and Liu Zeyuan \\ Beijing Institute of Spacecraft Environment Engineering, YouYi Road, Beijing, China
}

\begin{abstract}
Temperature measurement is a very important measurement project in spacecraft vacuum thermal test, and thermocouple measurement system is generally used for temperature measurement. In order to reduce the number of wire measuring circuit and improving the measurement system of anti disturbance and high measurement accuracy in vacuum thermal test process, which can be used in digital temperature measurement system of vacuum thermal test design, realizes the digital temperature measuring equipment of spacecraft and ground equipment. The system is composed of digital temperature sensor DS18B20 and acquisition device. It can be connected with remote monitoring computer through LAN network to realize remote monitoring of temperature. The hardware structure, communication protocol and software design of the digital temperature measurement system are given in this paper. The vacuum thermal environment test and comparison with the platinum resistance temperature measurement system. The results show that the stability of the system is good and the difference between the measured value and the PT100 measurement is within $0.5{ }^{\circ} \mathrm{C}$, and the linearity is about $\pm 0.1 \%$ which satisfies the requirement of vacuum thermal test.
\end{abstract}

\section{INTRODUCTION}

Vacuum thermal testing is a very important ground test that a spacecraft must pass before launching. In the spacecraft vacuum thermal test process, the important parameters that need to be measured are the temperature of the relevant parts of the test equipment and spacecraft [1].

Traditional spacecraft vacuum thermal test temperature measurement system generally uses thermocouple temperature measurement system. There are too many thermocouple cables passing through the spacecraft, which may change the original temperature distribution of the spacecraft to some extent ${ }^{[2]}$. Secondly, a thermocouple is an analog temperature sensor. Since the thermocouple cable between the measurement point and the signal processing unit is relatively long, the temperature gradient and noise of the environment through which the transmission path passes will adversely affect the measurement of high accuracy. And thermocouple wire thinner, longer, easy to be broken in the test preparation process or insulation damage, resulting in the test point circuit and insulation performance is not good, and the thermocouple temperature measurement system there are a large number of cables, paste and Welding process is complex, can not be used repeatedly and other shortcomings.
In view of the limitations of the thermocouple measurement system in spacecraft and ground tooling in the vacuum thermal experiment, a set of digital temperature measurement system was developed through the research on the digital measurement method and technology based on single bus. The digital temperature sensor technology used in spacecraft and ground equipment temperature measurement, the measurement line layout is simple (from the vacuum vessel to the outside of the vacuum vessel to use only three measuring leads), and the temperature sensor can be reused to solve the vacuum Thermal test thermocouple sensor caused by a one-time waste and paste in the test preparation, welding thermocouple complicated problems, but also increase the vacuum thermal test temperature measurement means diversity.

\section{SYSTEM DESIGN}

\subsection{Overall structure}

The overall hardware connection of the system is shown as in Fig. 1, mainly made up of the following parts: digital temperature sensor (DS18B20), splitter, acquisition equipment, switch, server and monitoring computer(PC).

\footnotetext{
*Corresponding author: zx cast@sina.com
} 


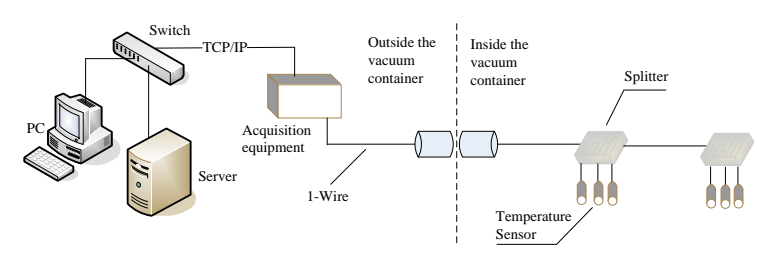

Figure 1 System hardware connection diagram

Acquisition devices receive the digital signal sent by the digital temperature sensor DS18B20 over and convert it to temperature data. All temperature sensors are mounted on a single bus through the splitter, thus reducing the number of measurement leads. PC monitors and manages the whole temperature measurement process, including setting of system parameters, touring temperature at each temperature measurement point, displaying temperature information in real time, and temperature alarm exceeding the upper and lower limits.

\subsection{Hardware and software components}

Acquisition devices are at the heart of the system, and their primary function is to control temperature sensors to collect temperature, store and display temperature data, and communicate with the monitoring computer over the TCP / IP protocol. Its main EMB3680 embedded industrial control board, C8051F020 microcontroller and their peripheral circuits, the block diagram shown in Figure 2.

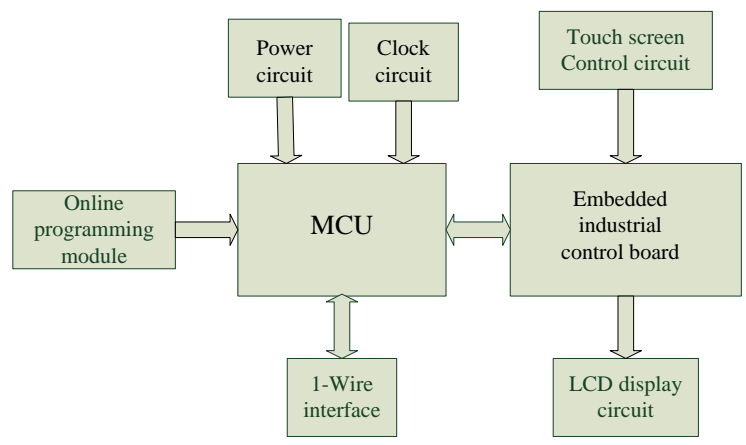

Figure 2 capture device block diagram

The system software design is based on XPE (Windows XP Embedded) platform, the use of Visual Basic language development. Windows XP Embedded is an embedded operating system that provides Windows operating system functionality in a modular form. Windows XP Embedded is based on the Win32 programming model and can be used to develop the required applications using common tools such as Visual Studio so that the embedded operating system integrates seamlessly with desktop applications and helps reduce development time.

PC software is running on the embedded IPC board temperature measurement system management software, which is responsible for monitoring and management of all temperature measurement points, including the following aspects:
(1) Data Acquisition: communication with the microcontroller and the monitoring computer, to achieve temperature data collection.

(2) Data Display: Display the temperature of all the temperature measurement points on the panel.

(3) Data storage: real-time temperature data stored in the local and server text files for monitoring program reads.

(4) Curve shows: allows users to the measured data, historical data to view the curve.

(5) Parameter Setting: Allows the user to set the parameters such as the measurement period of the system and the file saving path.

(6) Sensor Settings: The temperature sensor's address can be read and numbered.

The six aspects of the content can be divided into three functional modules, namely data acquisition and processing module, data management module and parameter setting module. Figure 3 shows the functional hierarchy of the management software for the digital temperature measurement system.

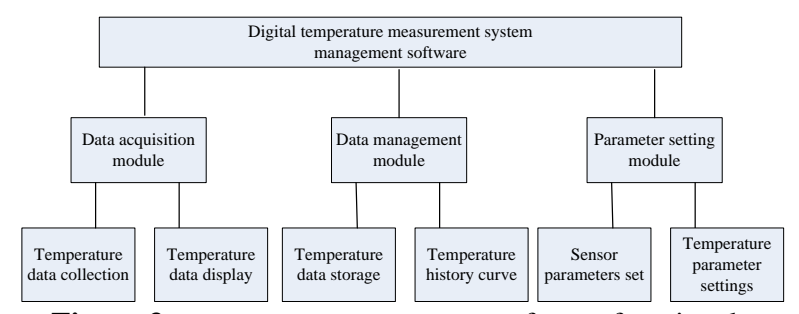

Figure 3 temperature management software functional hierarchy diagram

\section{SYSTEM HARDWARE DESIGN}

Acquisition instrument to EMB3680 embedded industrial control board and C8051F020 microcontroller as the core, to achieve temperature data acquisition, storage and acquisition instruments and computers for communication and other functions, and the use of liquid crystal display and touch screen to complete the manmachine interface.

\subsection{Embedded industrial control board}

The system adopts EMB3680 embedded industrial control board, which is equipped with XPE embedded operating system, which can be used as server for temperature data acquisition and remote PC can realize the function of accessing and data sharing through TCP / IP protocol. 7-inch touch LCD monitor can be its operation, to achieve human-computer interaction. Embedded industrial control board and microcontroller interface between the RS232.

\subsection{Acquisition equipment}

In this system using Silicon Labs C8051F020 microcontroller produced as a system control manager. The C8051F020 is a highly integrated single-chip, system-on-a-chip mixed-signal chip in a 100-pin package. 
The system uses a C8051F020 microcontroller as a control manager is mainly based on the following features:

(1) Has a JTAG interface, easy to burn and modify. Through the JTAG interface to achieve Flash, Fuse and lock the bit programming, you can easily program the burning and modification.

(2) Power-on reset and programmable brown-out detection. It has a calibrated $\mathrm{RC}$ oscillator with a clock frequency that can be selected by software.

(3) Rich I/O port: with 8 8-bit I/O port line. The greater the number of $\mathrm{I} / \mathrm{O}$ ports, the greater the number of single busses to which the temperature sensor can be attached and the efficiency of the temperature measurement during the vacuum thermal test.

(4) Has a programmable serial port USART: can easily communicate with the host computer through RS232 or RS485.

Another reason for choosing the C8051F020 microcontroller as a control manager is that it also has a JTAG interface that allows you to easily set breakpoints in your program and debug your software online. In the early stages of hardware and software debugging, you can save a lot of manpower, material and financial resources, greatly reducing the development cycle. The digital temperature measurement system described in this article uses the C8051F020 microcontroller UART0 serial communication interface, the corresponding pin is P0.0 and P0.1, through the RS232 communication with EMB3680 embedded industrial control board to communicate $^{[3]}$. The corresponding pin of single bus is $\mathrm{P} 1.4$; the JTGA programming chip is connected with the corresponding pin of $\mathrm{C} 8051 \mathrm{~F} 020 ; 24 \mathrm{MHZ}$ crystal oscillator is used to provide the clock circuit for the system and the decoupling capacitor is added in the circuit to improve the stability of the circuit. MCU circuit is shown in Figure 4.

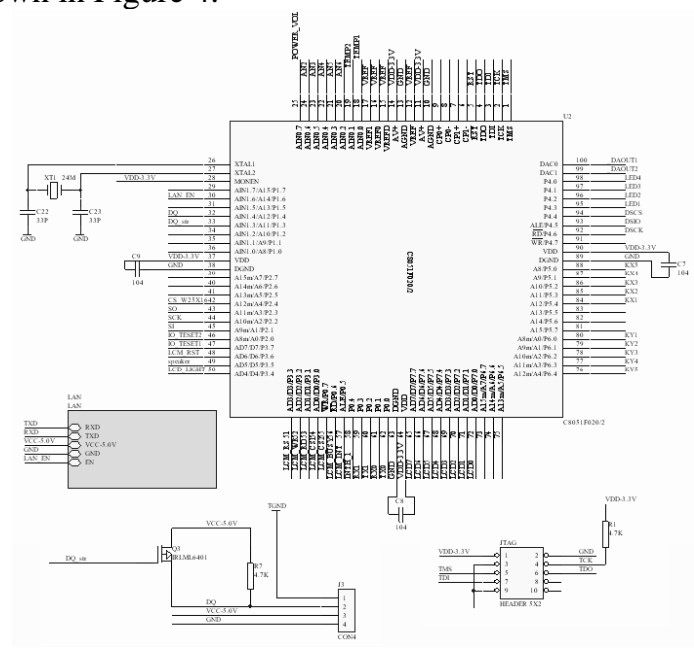

Figure 4 MCU application circuit

\subsection{Acquisition equipment}

The system temperature acquisition module consists of temperature sensor DS18B20 composition, DS18B20 has three pins, two of which are the power line VDD and ground line GND, the other one for the bus DQ. Available in two packages: 3-pin TO-92 package and 8pin SOIC package. Because TO-92 package form DS18B20 smaller, so the system uses TO-92 package temperature sensor.DS18B20 input and output are digital signals and compatible with the TTL level, so you can connect directly with the microcontroller interface. The system uses the temperature acquisition module shown in Figure 5. In the picture, one-chip computer PA0 mouth connects the single main line, can connect a plurality of DS18B20 on single bus line, adopt a $4.7 \mathrm{~K}$ pull-up resistor on the single bus line, makes the single bus line all while in idle state. In order to provide adequate operating current DS18B20, the system uses external power supply. In the practical application, the number of measurement channels can be determined according to the distribution of temperature measurement points of relevant parts on the spacecraft. The data bus can be led out from each I/O port of the single chip microcomputer, constitute a star network temperature measurement system.

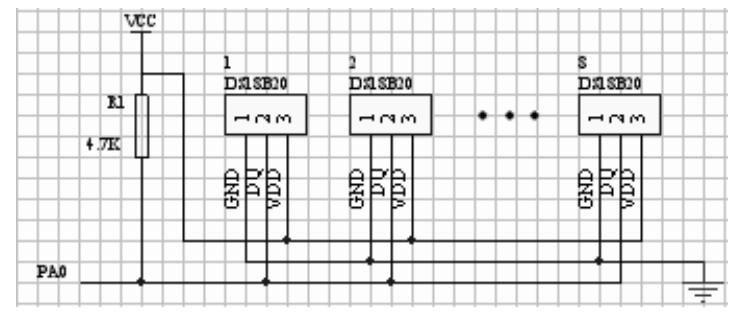

Figure 5 temperature acquisition module circuit

\subsection{LCD display and touch screen module}

The digital temperature measurement system interpersonal interface mainly by LCD (liquid crystal display) and touch screen to complete. The LCD controller selected in this paper is the SED1335 series chip made by Japan's SEIKO EPSON Corporation. SED1335 series chip instruction functions and interface with the CPU is relatively simple and easy to control, its drive capacity up to $640 \times 256$ dot matrix. LCD display shown in Figure 6.

Touch screen control chip is selected ADS7843, the chip includes two functions: The first is to complete the voltage switching circuit; The second is to collect the voltage at the contact point. The ADS7843 features 12bit A / D conversion and low on-resistance of its analog switches, making it easy to switch the voltage in the circuit and enabling fast analog-to-digital conversion. 


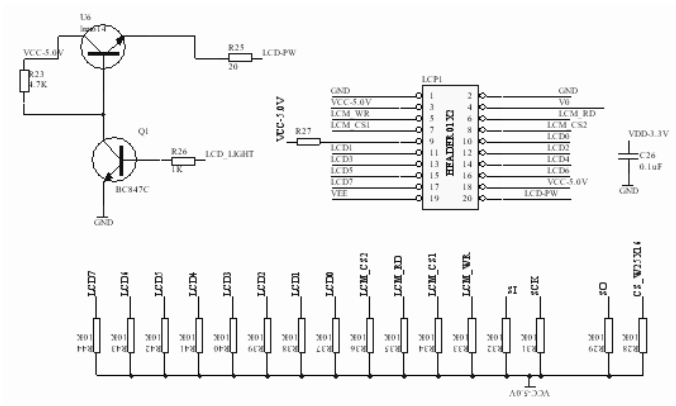

Figure 6 LCD display circuit

\section{ACQUISITION AND HOST SOFTWARE}

\subsection{Acquisition software design}

Acquisition software's main function is to achieve communication between the microcontroller and the temperature sensor. Acquisition software modular approach to write, mainly by the main program, the temperature acquisition instructions to receive and send subroutines, serial communication subroutines and other components $^{[4]}$. At the beginning of the temperature acquisition, the microcontroller sends a reset signal to the DS18B20 first through a single bus. After receiving the reset signal, the DS18B20 returns a successful reset signal to the microcontroller. The microcontroller then sends a start temperature conversion command to the DS18B20 with a delay of $800 \mathrm{~ms}$, waiting for the DS18B20 temperature conversion to complete. Finally, the microcontroller sends a read register data instruction to the DS18B20 to complete the entire temperature data acquisition process ${ }^{[5]}$.

It is worth noting that each DS18B20 ROM has a unique 64-bit serial number, the acquisition software by matching the 64-bit DS18B20 serial number, the match is read, the temperature of the sensor. Then match the next DS18B20 until all sensor temperatures have been read ${ }^{[6]}$. Before temperature acquisition begins, read the 64-bit serial number of the digital temperature sensor DS18B20 in ROM. Each DS18B20 has a 64-bit serial number that is different. If the number of DS18B20s used in the temperature measurement system is greater than or equal to 2 , write a software that reads the 64-bit serial number in the DS18B20 before temperature acquisition Use this serial number as a unique number in the temperature measurement system. It should be noted that, in reading the DS18B20 64-bit serial number, the bus last only one DS18B20. This work can be done before the vacuum thermal test.

In the process of software preparation, if the program is not running properly, you can use the virtual oscilloscope to observe the timing of the DS18B20 work, through the timing analysis to modify the program. Oscilloscope display timing diagram shown in Figure 7. DS18B20 data read and write, including read "0", read "1", write "0", write "1" four types of write timing in
Figure 6 is written to the DS18B20 "11001100" The timing is read from the DS18B20 "11011100" (read from the lower).

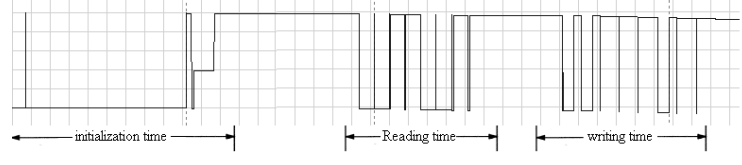

Figure 7 DS18B20 timing diagram

\subsection{Software Main Interface}

The main interface of the system software shown in Figure 8 , respectively by three modules, including the measurement interface, the probe settings and instructions The main functions of the measurement interface module are as follows: (1) sampling the enabled temperature sensor; (2) displaying the temperature of all the measuring points; and (3) saving the historical temperature data. Click "Measurement Interface", the software interface switch to as shown in Figure 9, only the first seven temperature sensors are enabled. This screen shows the temperature of 50 measurement points. If a measurement point is not enabled, "Null" is displayed. When you click the temperature value of a measurement point on the measurement interface, the historical curve of the temperature change of the measurement point will be displayed.

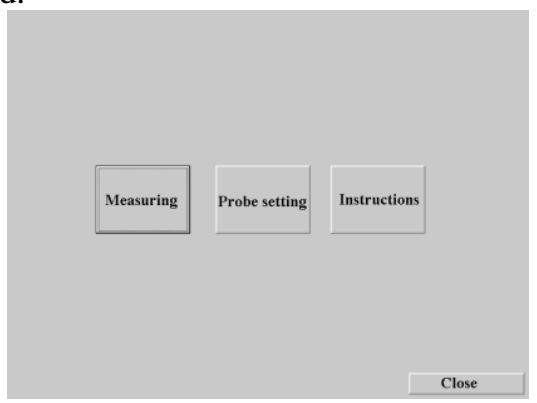

Figure 8 test software main interface

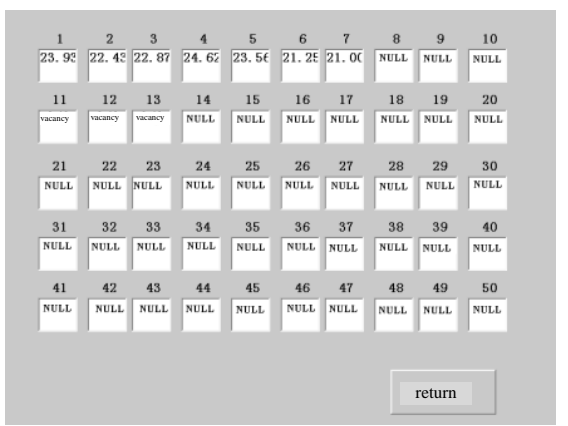

Figure 9 measurement interface display

\section{SYSTEM TESTING AND ANALYSIS}

\subsection{Comparison of test methods}

In order to verify the accuracy of the system to measure the temperature and linearity indicators, digital temperature measurement system was tested. The test 
was carried out in space environment simulator of Beijing Institute of Satellite Environmental Engineering. The selected calibration device was a copper cylinder with a diameter of $100 \mathrm{~mm}$ and a height of $100 \mathrm{~mm}$, as shown in Figure 10. A hole with a diameter of $40 \mathrm{~mm}$ and a height of $40 \mathrm{~mm}$ was drilled in the middle of the cylinder.

The thermal inertia of the specimen is relatively large, and the cavity can form a temperature field with a relatively uniform temperature. Place a Pt100 and two DS18B20 temperature sensors in the inner cavity, then inject vacuum thermal grease. It can be considered that the temperature field of each temperature sensor in the cavity is the same.

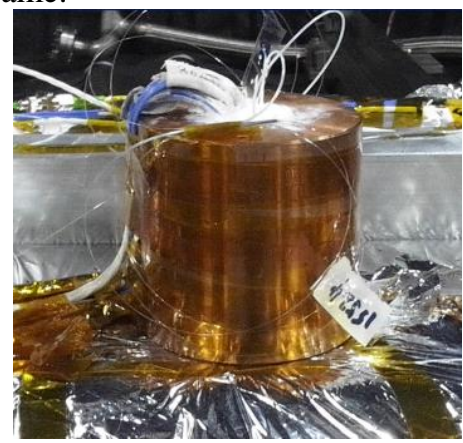

Figure 10 The calibration device

\subsection{Error Analysis}

Due to the large amount of data, we selected some of the temperature data for analysis and selected the test data from normal temperature to $-40{ }^{\circ} \mathrm{C}$. The measured temperature of each temperature sensor are shown in Table 1.

Table 1 Temperature data of each sensor

\begin{tabular}{|c|c|c|}
\hline Pt100/ $\left({ }^{\circ} \mathrm{C}\right)$ & 1\#DS18B20 & 2\#DS18B20 \\
\hline-20.37 & -20.063 & -20.125 \\
\hline-10.572 & -10.25 & -10.188 \\
\hline-0.281 & 0 & 0.125 \\
\hline 10.104 & 10.375 & 10.363 \\
\hline 21.372 & 21.563 & 21.75 \\
\hline 40.534 & 40.75 & 40.875 \\
\hline 61.381 & 61.563 & 61.75 \\
\hline 81.212 & 81.5 & 81.588 \\
\hline
\end{tabular}

In this test system, platinum resistance has been accurately calibrated, and its measurement error is less than $\pm 0.1{ }^{\circ} \mathrm{C}$, so the temperature measured by the platinum resistance temperature measurement system as a true value, DS18B20 measured temperature value is measured.

According to the data in Table 1, the temperature error curve between the digital temperature measurement system and the platinum resistance temperature measurement system can be obtained. As can be seen from Figure 11, the difference between the digital temperature measurement system and the platinum resistance temperature measurement system is within $0.5{ }^{\circ} \mathrm{C}$. Both error 1 and error 2 are positive, due to the thermal inertia of the digital temperature sensor
DS18B20 that is greater than the thermal inertia of the PT100.

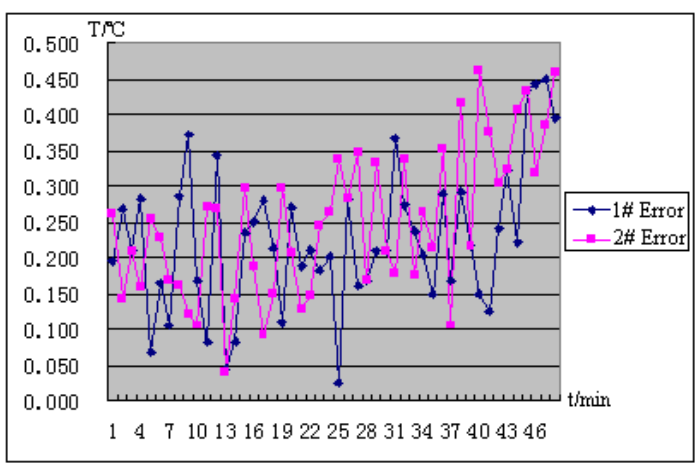

Figure 11 Error curve

\subsection{Linearity Analysis}

DS18B20 measurement range of $-55^{\circ} \mathrm{C} \sim+125{ }^{\circ} \mathrm{C}$, the normal measurement of the full scale of $50 \%$ to $70 \%$. In order to ensure the measurement accuracy of DS18B20, taking the temperature data in the range of $-10{ }^{\circ} \mathrm{C} \sim+$ $80{ }^{\circ} \mathrm{C}$, we found that the maximum deviation of the calibration curve and fitting curve appears in the temperature range of $-10{ }^{\circ} \mathrm{C} \sim 0{ }^{\circ} \mathrm{C}$, as shown in Figure 12 Show.

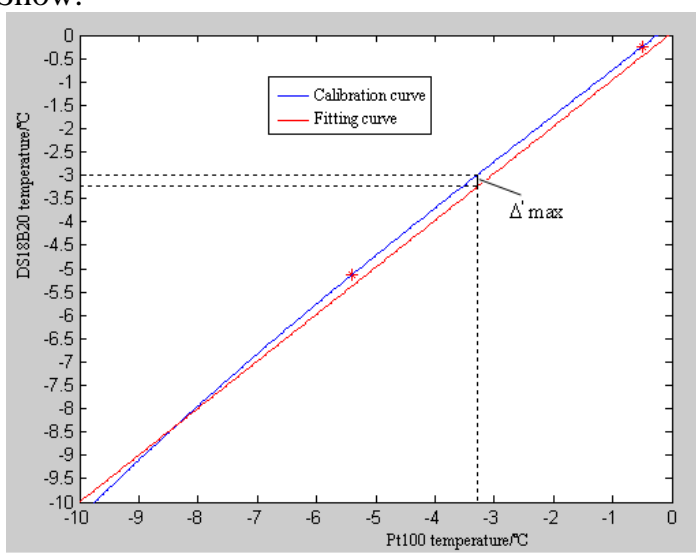

Figure 12 Digital temperature measurement system linearity curve

By linearity calculation formula available:

$$
\delta_{f}= \pm \frac{0.25}{125-(-55)} \times 100 \%= \pm 0.139 \%
$$

It can be seen from the calculation results, digital temperature measurement system has a very good linearity.

\section{CONCLUSIONS}

This paper introduces a digital temperature measurement system that can be used for vacuum thermal test. The temperature sensor of this measurement system has thick leads, which are hard to be damaged and broken. The temperature sensor can be reused. The system has the advantages of simple structure, easy operation and easy to carry specialty. And the sensor used in the system is a single-bus digital temperature sensor. All the temperature sensors need only be mounted on one measuring cable, and the anti-interference ability of the digital signal is 
strong, so it is more suitable for comparing the number of measuring cables and the testing environment Bad occasion. The test and analysis of the symbol system show that the measurement error is better than $\pm 0.5{ }^{\circ} \mathrm{C}$ and the linearity is about $\pm 0.1 \%$ under vacuum thermal environment. The digital temperature measurement system is not only suitable for the temperature measurement of spacecraft groundworking equipment, but also suitable for the temperature measurement of the components of the vacuum thermal test section and the temperature measurement of the outdoor spacecraft box and so on, and has a wide application prospect.

\section{ACKNOWLEDGEMENTS}

The work is supported by key project of the advance research field fund provided by the Ministry of Equipment Development (NO. 6140923020301).

\section{REFERENCES}

1. Guo G. 2009. Temperature measure system for spacecraft thermal vacuum tests[J]. Spacecraft Environment Engineering.

2. Liu Q, Huang X . 2000. The new type sensors and their application in signal detection ICSC2000[C].

3. Zhong W, Wang Y. 2015. Mechanism Research of Serial Port Communication in Basis and Relevant Realization[J]. Computer Engineering.

4. LIU B. 2015. DS1820 Sensor-based Monobus Multi point Temperature Measurement Technology[J]. MACHINERY \& ELECTRONICS.

5. Deng J, Yang B, Li W etc. 2014. Realization of the industrial gateway Based on DS18B20[J]. Control \& Automation.

6. Zhong W, Wang Y. 2015. Mechanism Research of Serial Port Communication in Basis and Relevant Realization[J]. Computer Engineering. 